\title{
Establishment and evaluation of a Beagle model of grade III pancreatic trauma
}

\author{
HANJING GAO ${ }^{1,2^{*}}$, QING SONG ${ }^{3 *}$, FAQIN LV ${ }^{1}, \mathrm{BIN} \mathrm{SHI}^{4}$, PENGFEI WANG $^{4}$,

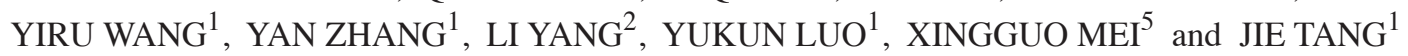 \\ ${ }^{1}$ Department of Ultrasound, Chinese PLA General Hospital, Beijing 100853; ${ }^{2}$ Department of Ultrasound, \\ 161st Hospital of Chinese PLA, Wuhan, Hubei 430010; ${ }^{3}$ Department of Ultrasound, General Hospital of Beijing Military \\ Region, Beijing 100700; ${ }^{4}$ Department of Hepatobiliary Surgery, Chinese PLA General Hospital, Beijing 100853; \\ ${ }^{5}$ Department of Pharmaceutics, Beijing Institute of Pharmacology and Toxicology, Beijing 100039, P.R. China
}

Received October 23, 2015; Accepted February 24, 2017

DOI: $10.3892 /$ etm.2018.6656

\begin{abstract}
Pancreatic trauma (PT) is a severe abdominal injury that is often combined with multiple organ injury. It is a severe disease that is difficult to diagnose and has a high mortality rate, particularly for grade III PT. The pathogenesis, disease progress and complications have not been fully investigated due to the lack of a reliable animal model. To address this, a Beagle model of grade III PT was established in the present study using a procedure involving rupture of the main pancreatic duct. Peripancreatic effusions and the degree of pancreatic damage were examined by routine ultrasound and contrast-enhanced ultrasound (CEUS). Also, ascites were collected for the examination of amylase and lipase levels, and whole blood samples were collected for the analysis of amylase, lipase, C-reactive protein (CRP), interleukin (IL)-6 and tumor necrosis factor (TNF)- $\alpha$ levels in the serum. Urine samples were also collected for the examination of trypsinogen activation peptide (TAP). In addition, the pancreas was sectioned and stained with hematoxylin and eosin. In comparison with routine ultrasound, CEUS showed a large area of focal trauma, with a depth greater than half of the anteroposterior diameter of the pancreas, with a clear boundary, clear capsular rupture and trauma induced by active bleeding. The volume of ascites peaked at $48 \mathrm{~h}$ post-trauma and decreased thereafter. Amylase and lipase levels in the ascites were elevated at $24 \mathrm{~h}$ post-trauma and significantly decreased at 48 and $72 \mathrm{~h}$ post-trauma $(\mathrm{P}<0.01)$. In addition, serum amylase and lipase levels increased to peak levels at $48 \mathrm{~h}$ post-trauma
\end{abstract}

Correspondence to: Dr Jie Tang, Department of Ultrasound, Chinese PLA General Hospital, 28 Fuxing Road, Beijing 100853, P.R. China

E-mail: txiner@vip.sina.com

${ }^{*}$ Contributed equally

Key words: grade III pancreatic trauma, ultrasound contrast, Beagle, model and then decreased $(\mathrm{P}<0.05)$, while serum CRP, IL-6 and TNF- $\alpha$ levels peaked at $24 \mathrm{~h}$ post-trauma and then decreased $(\mathrm{P}<0.05)$. Urinary TAP levels also peaked at $24 \mathrm{~h}$ post-trauma and subsequently decreased $(\mathrm{P}<0.05)$. At $72 \mathrm{~h}$ post-trauma, the pancreatic cells were loosely distributed, with damaged acini, hyperchromatic nuclei and severe inflammatory cell invasion. These results indicated that the Beagle model of grade III PT was satisfactorily established, and that CEUS is potentially useful as an auxiliary diagnosis method for PT. This animal model may be useful for studying the pathogenesis, disease progress and complications of PT.

\section{Introduction}

The pancreas is located deep in the retroperitoneal space, and has significant endocrine and exocrine functions $(1,2)$. When the pancreas is damaged, the clinical symptoms are often overshadowed by symptoms and signs of other organs, which may cause a delay in its diagnosis and treatment, and often induces severe complications, such as traumatic pancreatitis, pancreatic abscess, pseudocyst and pancreatic fistula (3). As the popularity of expressways and motor vehicles increases, and with the rapid development of construction and other types of industry, the occurrence of accidents has increased the morbidity of pancreatic trauma (PT) (4). It has been reported that the morbidity of PT is $\sim 45 \%$, and the mortality of PT is typically $10-30 \%$, even reaching $60 \%$ when diagnosis and treatment are delayed $(5,6)$. Therefore, PT is one of the most intractable diseases of abdominal trauma, and is the subject of considerable attention. However, due to the particular characteristics of the anatomical site and physiological functions of the pancreas, the diversity of PT injury factors, and the lack of effective drug treatments and classical animal models, the study of PT is challenging and is lagging behind that of other abdominal parenchymal organs, such as the liver and spleen. The lack of a classical animal model is particularly limiting to the study of PT.

A few reliable animal models of PT have been reported, that have been prepared using various experimental approaches to mimic clinical PT. In one model, a BIM-III multifunctional biological impact machine was used to establish a rat PT model, 
in which bloody ascites were observed, in addition to increased amylase levels in the serum and ascites, and hyperemia, edema and necrosis of the pancreas (7). In another model, following the sharp separation of the distal pancreas and hemostasis with minimal cauterization, a pig PT model was established that exhibited severe inflammation, fibrosis and necrosis (8). In a third model, the pancreas was squeezed in front of the spine using hemostatic forceps to establish a minipig model with various degrees of PT, and increased levels of amylase and lipase in the serum (9). Although these models reflect the disease symptoms of PT, they have certain limitations, such as model instability, difficult procedures, unsatisfactory levels of damage and poor clinical applicability.

Beagle dogs were selected as experimental subjects in the present study in view of the anatomical structure and physiological functions of dogs being much more closer to those of humans than are those of pigs and rats, in addition to these dogs having a docile temperament, good compliance and being easy to experiment on (10). Theoretically, ultrasound examination should provide good imaging results due to the relatively thin abdominal walls of dogs. However, the ability of ultrasound to display the pancreas is poor for dogs, and the pancreatic echo is not clearly distinguishable. The dog thorax is quite deep, and the pancreas is freely located in the abdominal cavity, with its left lobe positioned deeply and covered by the liver and stomach at the front, and so body surface ultrasound is not able to detect it. In addition, the right lobe of the pancreas is deeply located in the intestinal cavity in close dorsal proximity to the duodenum, and is readily shielded by the intestinal canal and disturbed by gas, and body surface ultrasound is also not able to detect it. In the present study, the duodenum intestinal wall was fixed to the abdominal wall making the right lobe move closer to the abdominal wall to avoid shielding of the thorax and the disturbing effects of gas in the intestinal tract, and thereby to improve the display rate and image quality of the pancreas during ultrasound examination.

\section{Materials and methods}

Experimental animals. In total, 5 female Beagle dogs (weight, 10-12 kg and 23-24 months of age) were provided by the National Institutes of Health for the Care of Laboratory Animals [license number, SCXK (Beijing, China) 2011-0003]. The dogs were housed at a temperature of $24-28^{\circ} \mathrm{C}$, humidity of $45-60 \%$ and a light-dark cycle of $12-\mathrm{h}$ with ad libitum access to food and water.

Establishment of a Beagle model of grade III PT. After fasting for $18 \mathrm{~h}$, the Beagles were anesthetized with a forelimb injection of pentobarbital sodium (3\%; Sigma-Aldrich; Merck KGaA, Darmstadt, Germany) at a preoperative dose of $30 \mathrm{mg} / \mathrm{kg}$, and then fixed on an operating table to perform endotracheal intubation. A gastric tube was inserted and linked to an apparatus for vacuum aspiration and gastrointestinal decompression. Heparin (300 IU/kg; Sigma-Aldrich; Merck KGaA) was then administered by intravenous injection followed by intraoperative anesthetization with $3 \%$ pentobarbital sodium at a dose of $3 \mathrm{mg} / \mathrm{kg}$.

When the Beagles were anesthetized, the abdominal hair was removed by shaving, and the skin was sterilized prior to a medial incision being made to expose the pancreatic tissue through layered laparotomy. Subsequently, focal parenchyma trauma combined with main pancreatic duct damage, where the pancreatic parenchyma was distanced $7 \mathrm{~cm}$ from the pylorus, was created using hemostatic forceps, and the depth of the focus was half of the anteroposterior diameter of the pancreas. After this, the incision was sutured layer by layer, using a needle and 4-0 Mousse thread with a needle layer by layer, and an intravenous injection of antibiotic was subsequently administered. According to the Organ Scaling Committee of the American Association for the Surgery of Trauma, pancreatic damage with rupture of the main pancreatic duct may be used to mimic grade III PT (11). The study protocol was approved by the Chinese People's Liberation Army General Hospital Ethics Committee (Beijing, China).

Routine ultrasound and contrast-enhanced ultrasound (CEUS) examination. Following the modeling of PT, the focus location, scope, shape, boundary and internal echo were observed immediately using routine ultrasound. Subsequently, CEUS examination of the abdomen was performed using a color Doppler diagnostic apparatus (CX50; Philips Healthcare, DA Best, The Netherlands; probe, L12-3, 3-12 MHz) as follows. The probe was placed on the right side of the abdomen and the inside of the abdominal wall, and the device condition was set to provide abdominal contrast imaging over time. Simultaneously, $0.5 \mathrm{ml}$ SonoVue (Bracco S.p.A., Milan, Italy) was injected by an intravenous bolus injection technique, followed by fast flushing of the injection tubing with $5.0 \mathrm{ml}$ normal saline. Subsequently, dynamic images of the pancreas were recorded through continuous examination to observe the focal trauma location, scope, shape and boundary, and to determine whether or not contrast agent filling, retention and overflow occurred. If the procedure was repeated, the interval time between examinations was $>10 \mathrm{~min}$. The probe was isolated by a sterile protective membrane under sterile conditions.

After reviving the Beagles, $1.0 \mathrm{ml}$ bucinnazine hydrochloride injection (100 mg/2 ml; Shenyang First Pharmaceutical Co., Ltd., Shenyang, China) was injected into the coxal muscle. When the vital signs were stable and no bleeding was observed, the Beagles were each returned to a single cage, and fasted for $18 \mathrm{~h}$ with continuous observation for 3 days. In addition, the general condition and survival counts of the dogs were recorded at $0.5 \mathrm{~h}$ pre-surgery, and 24,48 and $72 \mathrm{~h}$ post-surgery, and samples of ascites, whole blood, urine and pancreatic tissues were collected during surgery and used for subsequent examinations.

Measurement of ascites amylase and lipase levels. At 24, 48 and $72 \mathrm{~h}$ post-surgery, ascites were collected and centrifuged at 7,200 x g for $30 \mathrm{~min}$ at room temperature. Following this, assays of amylase and lipase expression were conducted using a dog amylase ELISA kit (cat. no. C016) and dog lipase ELISA kit (cat. no. A054; both from Nanjing Jiancheng Bioengineering Institute, Nanjing, China). The data were recorded at $450 \mathrm{~nm}$ using a microplate reader (Bio-Rad Laboratories, Inc., Hercules, CA, USA) and analyzed using Origin 9.5 software (http://www.originlab.com/). 

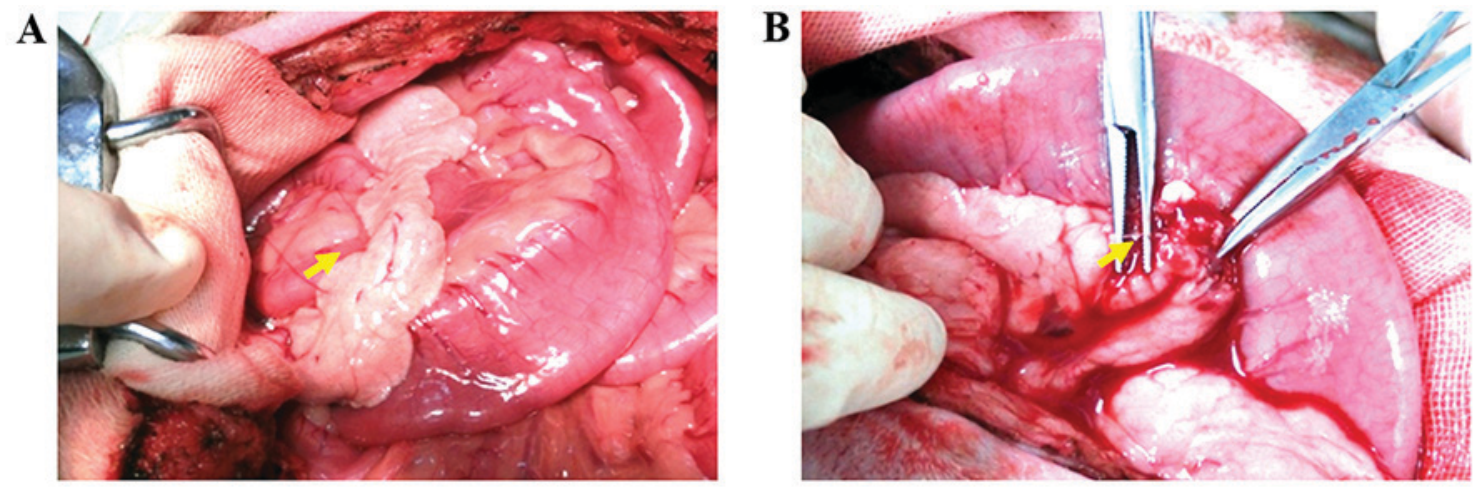

Figure 1. Anatomical images showing the main pancreatic duct prior to and during grade III pancreatic trauma induction in a Beagle dog. The yellow arrows indicate (A) the intact main pancreatic duct and (B) division of the main pancreatic duct.

Measurement of serum amylase, lipase, $C$-reactive protein (CRP), interleukin-6(IL-6) and tumornecrosisfactor- $\alpha(T N F-\alpha)$ levels. At $0.5 \mathrm{~h}$ pre-surgery, and 24, 48 and $72 \mathrm{~h}$ post-surgery, samples of whole blood were collected and centrifuged at $4,500 \mathrm{x}$ g for $30 \mathrm{~min}$ at room temperature to separate the serum for measurement of amylase, lipase, CRP, IL- 6 and TNF- $\alpha$ levels. For the amylase assay, a dog amylase ELISA kit (cat. no. C016) was used and results were recorded at $450 \mathrm{~nm}$ using a microplate reader. Similarly, dog lipase ELISA (cat. no. A054; both from Nanjing Jiancheng Bioengineering Institute), and dog CRP ELISA (cat. no. F28110), dog IL-6 ELISA (cat. no. F28180) and $\operatorname{dog}$ TNF- $\alpha$ ELISA kits (cat. no. F28330; all from Shanghai Westang Bio-Tech Co., Ltd., Shanghai, China) were used and results were recorded at $450 \mathrm{~nm}$ using microplate reader. The assays were conducted according to the manufacturers' instructions, and data were analyzed using Origin 9.5 software.

Measurement of urinary trypsinogen activation peptide (TAP) levels. At $0.5 \mathrm{~h}$ pre-surgery, and 24, 48 and $72 \mathrm{~h}$ post-surgery, urine samples were collected and centrifuged at 7,200 $\mathrm{x} \mathrm{g}$ for $30 \mathrm{~min}$ at room temperature followed by examination of the expression of TAP using a dog TAP ELISA kit (cat. no. CSB-E13461c; Wuhan Cusabio Biotech Co., Ltd., Wuhan, China). The data were recorded at $450 \mathrm{~nm}$ using a microplate reader and analyzed using Origin 9.5 software.

Hematoxylin and eosin $(H \& E)$ staining. At $72 \mathrm{~h}$ post-surgery, the surviving Beagles were sacrificed for examination of the changes induced in the pancreas. Thus, pancreatic tissues were collected and fixed with $10 \%$ paraformaldehyde prior to performing H\&E staining as previously described (12). Slides were deparaffinized and rehydrated, and frozen or vibratome sections were mounted on slides and rehydrated. The sections were slightly overstained with hematoxylin for 3-5 min, according to the thickness of the section (3-5 $\mu \mathrm{m})$ and fixed using $4 \%$ paraformaldehyde (for up to $20 \mathrm{~min}$ if the solution was not fully fixed), and excess stain was removed using tap water. The sections were differentiated and destained for a few sec in acidic alcohol until the sections appeared red, which typically took four or five dips. The sections were then briefly rinsed in tap water to remove the acid. Bicarbonate was applied for $\sim 2$ min until the nuclei were sharply visible in blue. The hematoxylin-stained slides from the final tap water rinse were placed in $70 \%$ ethanol for $3 \mathrm{~min}$ and then in eosin for $2 \mathrm{~min}$. Finally, the slides were subjected to three washes with $95 \%$ ethanol for 5 min and then transferred to the first absolute ethanol of the clearing series. Images were captured using a brightfield microscope connected to a CCD camera at $x 20$ and $\times 40$ magnification.

Statistical analysis. All data are expressed as the mean \pm standard deviation. Statistical analysis was performed with one-way analysis of variance using SPSS software (version 21.0; http://spss.en.softonic.com/; IBM SPSS, Armonk, NY, USA), and Student's t-tests were performed for comparisons of two groups. $\mathrm{P}<0.05$ was considered to indicate a statistically significant difference.

\section{Results}

Establishment of a Beagle model of grade III PT is verified by ultrasound and general condition. In order to establish the grade III PT model, the main pancreatic duct was exposed as indicated by the yellow arrow in Fig. 1A, and divided using hemostatic scissors as shown by the yellow arrow in Fig. 1B to correctly establish the grade III PT model. After modeling, all of Beagles were surviving, lethargic and laid passively and laterally at $6 \mathrm{~h}$, were slowly moving at $12 \mathrm{~h}$, and had begun to eat again at $24 \mathrm{~h}$, although they frequently vomited.

When examined by routine ultrasound, the pancreas was completely displayed, with locally uneven echo, an obscured boundary at the lower echo region and irregularly shaped PT (Fig. 2). As the time after the modeling surgery increased, the margins of the focal trauma tended to become smoother, and its shape remained irregular with lower or no echo (data not shown). CEUS detected a large region of focal trauma, with a depth greater than one-half of the anteroposterior diameter of the pancreas, with a clear boundary, clear capsular rupture and trauma from active bleeding (Fig. 2).

Amylase and lipase levels of ascites peak at $48 \mathrm{~h}$ and decrease at $72 \mathrm{~h}$ after $P T$. The volume of ascites detected by ultrasound was increased to a peak at $48 \mathrm{~h}$ after the PT modeling surgery and decreased at $72 \mathrm{~h}$ post-surgery (Table I). The expression of amylase and lipase in the ascites reached the highest levels at $24 \mathrm{~h}$ post-surgery, and then significantly decreased at 48 and $72 \mathrm{~h}$ post-surgery ( $\mathrm{P}<0.01$ vs. $24 \mathrm{~h}$ post-surgery; Fig. 3). At $72 \mathrm{~h}$ after the surgery, the expression of amylase and lipase in the ascites was $>3$-fold indicating pancreatic fistula. 


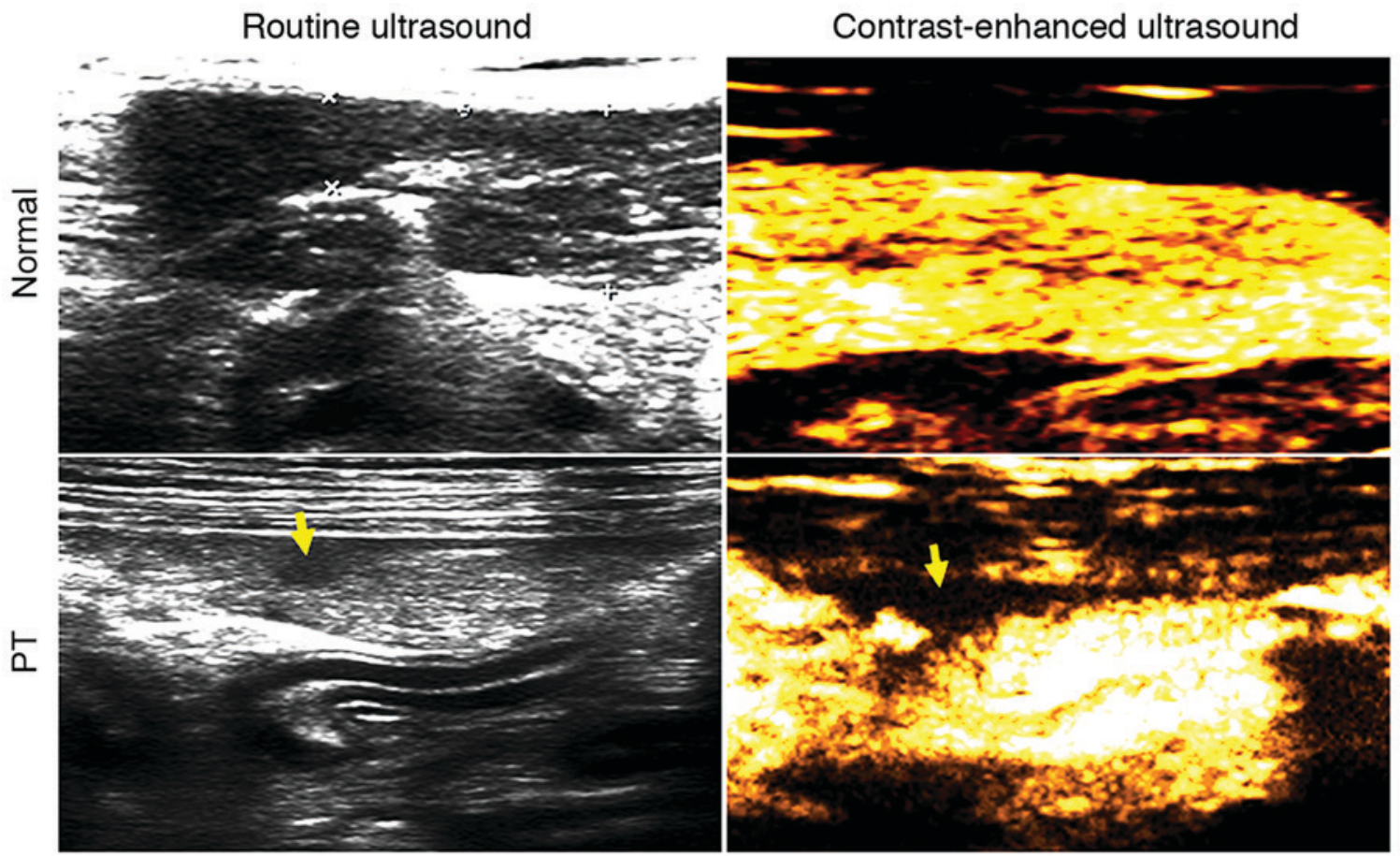

Figure 2. Pancreatic trauma examination by routine ultrasound and contrast-enhanced ultrasound. In the PT model, contrast-enhanced ultrasound exhibited extensive focal trauma, with a depth greater than half of the anteroposterior diameter of the pancreas with a clear boundary, clear capsular rupture and trauma induced active bleeding. PT, pancreatic trauma.

Table I. Volume of ascites detected by ultrasound in vivo.

\begin{tabular}{ll}
\hline Time post-surgery & Ascites dimensions $(\mathrm{cm})$ \\
\hline $24 \mathrm{~h}$ & $2.33 \times 0.6 \times 3.1$ \\
$48 \mathrm{~h}$ & $3.41 \times 0.91 \times 3.6$ \\
$72 \mathrm{~h}$ & $2.6 \times 1.8 \times 2.7$ \\
\hline
\end{tabular}

Serum amylase, lipase, CRP, IL-6 and TNF- $\alpha$ levels increase after $P T$ and then decrease. The expression levels of serum amylase and lipase increased after the PT modeling surgery, peaking at $48 \mathrm{~h}$ after the trauma to the pancreas and then decreasing $(\mathrm{P}<0.05$ vs. $0.5 \mathrm{~h}$ pre-surgery; Fig. $4 \mathrm{~A}$ and $\mathrm{B})$. The expression levels of serum CRP, IL- 6 and TNF- $\alpha$ were also increased after the PT modeling surgery, peaking at $24 \mathrm{~h}$ post-surgery and then decreasing $(\mathrm{P}<0.05$ vs. $0.5 \mathrm{~h}$ pre-surgery; Fig. 4C-E).

Urinary expression of TAP peaks at $24 \mathrm{~h}$ after PT and then decreases. The urinary expression of TAP was increased to a peak level at $24 \mathrm{~h}$ after the PT modeling surgery, and then decreased at 48 and $72 \mathrm{~h}$ post-surgery ( $\mathrm{P}<0.01$ vs. $0.5 \mathrm{~h}$ pre-surgery; Fig. 5 ).

PT models exhibit loosely distributed cells, with severely damaged acini, hyperchromatic nuclei and inflammatory cell invasion. The H\&E staining results demonstrate that in the pancreas of a normal Beagle dog, the pancreatic cells were tightly distributed, with regular acini, no hyperchromatic nuclei and no inflammatory cell invasion (Fig. 6). However, at $72 \mathrm{~h}$ after the trauma to the pancreas, pancreatic cells were loosely distributed, and displayed damaged acini, hyperchromatic nuclei and severe inflammatory cell invasion (Fig. 6).

\section{Discussion}

In this study, a Beagle model of grade III PT was established, which involved transection of the main pancreatic duct and pancreatic parenchyma, $7 \mathrm{~cm}$ distant from the pylorus. After modeling, the volume of ascites and the levels of amylase and lipase it contained were increased to peak levels at $24 \mathrm{~h}$ post-surgery and then decreased. Serum amylase and lipase levels were increased to their highest levels at $48 \mathrm{~h}$ post-surgery and then decreased, whereas serum CRP, IL- 6 and TNF- $\alpha$ levels peaked at $24 \mathrm{~h}$ post-surgery and then decreased. Urinary TAP levels were elevated in this model, peaking at $24 \mathrm{~h}$ post-surgery, and then decreased slightly at 48 and $72 \mathrm{~h}$ post-surgery. When compared with normal pancreatic cells, pancreatic tissue specimens examined $72 \mathrm{~h}$ after the induction of trauma exhibited loosely distributed pancreatic cells, with damaged acini, hyperchromatic nuclei and severe inflammatory cell invasion. All of these observations indicated that the Beagle model of grade III PT was correctly established, and was easy to prepare, with clear and reliable grading, good repeatability and controllability, and without damaging effects on the liver and intestinal canal.

Several imaging evaluation approaches for PT have been reported, including ultrasound, computed tomography (CT), endoscopic retrograde cholangiopancreatography (ERCP) and magnetic resonance cholangiopancreatography (MRCP) (13). Among these, ultrasound, CT and MRCP are noninvasive examination techniques. CT, particularly contrast-enhanced CT (CECT), has a high sensitivity and specificity, and is able 

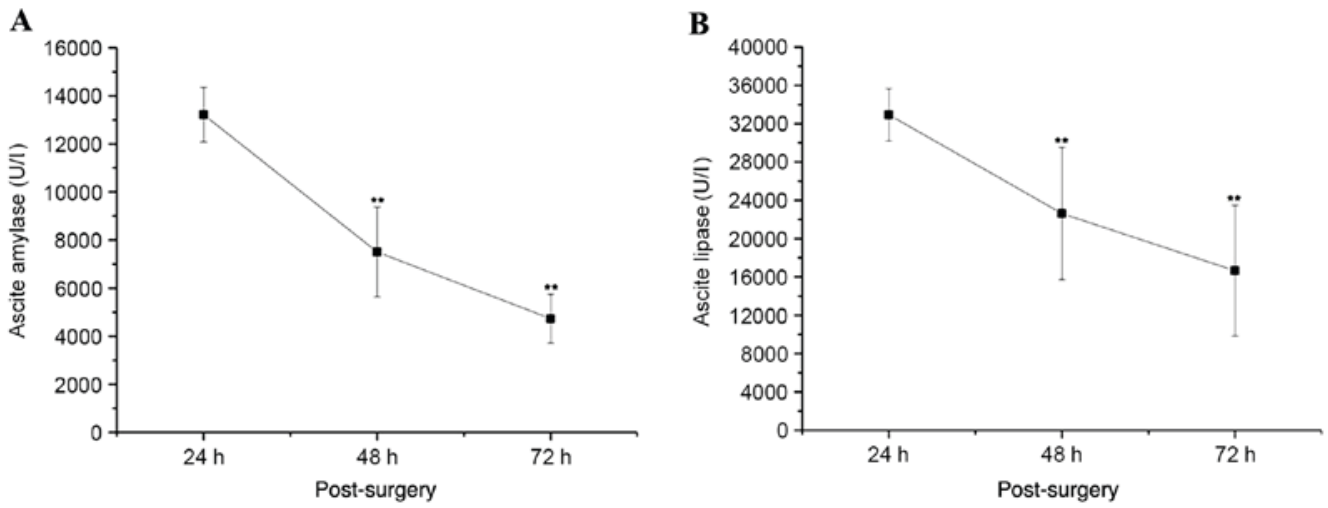

Figure 3. ELISA results for amylase and lipase in the ascites. (A) Amylase and (B) lipase levels in the ascites as detected by ELISA. The levels of amylase and lipase were elevated at $24 \mathrm{~h}$ after pancreatic trauma-inducing surgery, and were significantly decreased at 48 and $72 \mathrm{~h}\left({ }^{* *} \mathrm{P}<0.01 \mathrm{vs} .24 \mathrm{~h} ; \mathrm{n}=5\right)$.
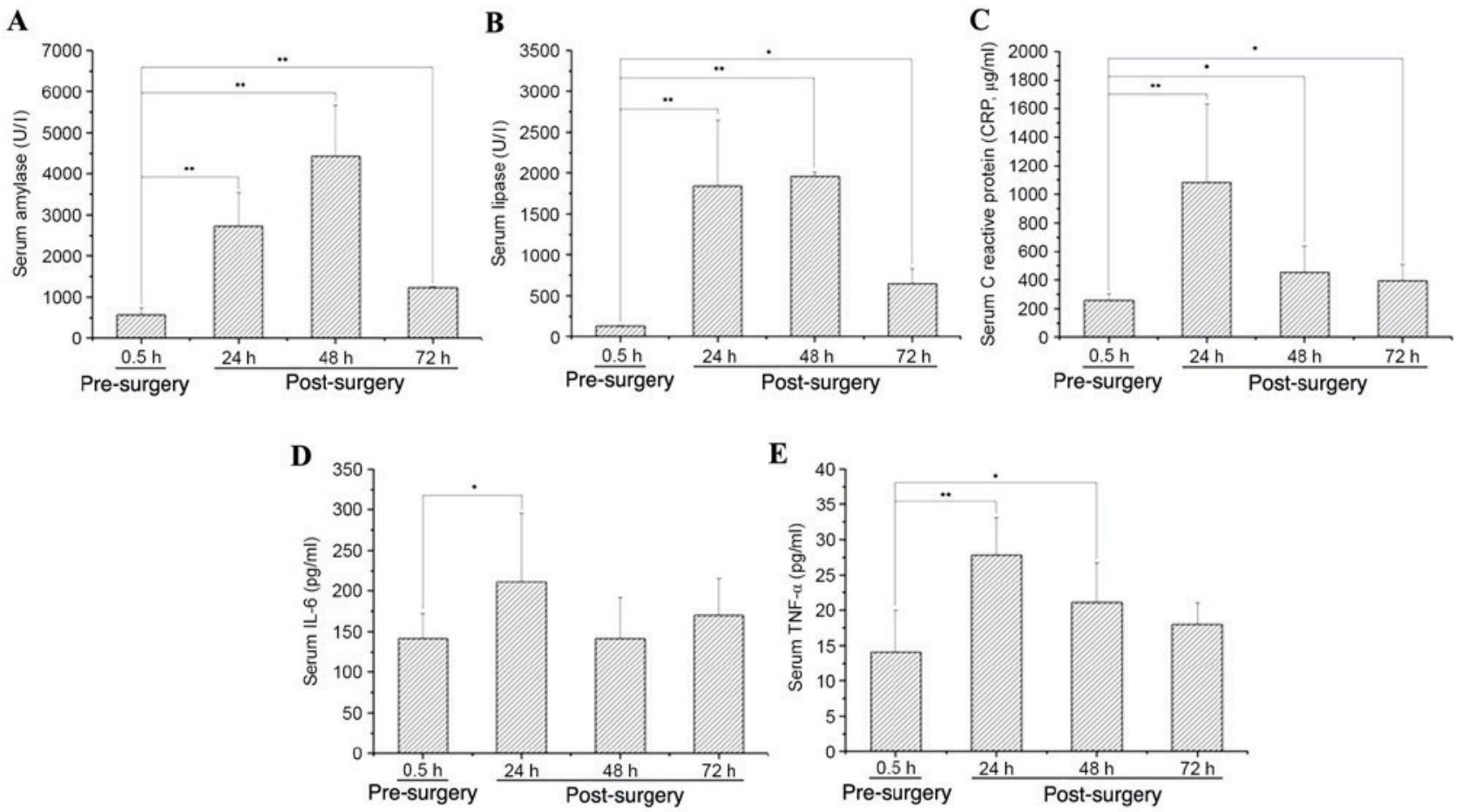

Figure 4. ELISA results for serum biochemical indices. Serum levels of (A) amylase, (B) lipase, (C) CRP, (D) IL-6 and (E) TNF- $\alpha$ as detected by ELISA. Serum amylase and lipase levels were increased after pancreatic trauma-inducing surgery, peaking at $48 \mathrm{~h}$ and then decreasing; serum CRP, IL-6 and TNF- $\alpha$ levels were also increased post-surgery, peaking at $24 \mathrm{~h}$ and then decreasing ( $\mathrm{P}<0.05$ and ${ }^{* *} \mathrm{P}<0.01$ vs. pre-surgery; $\left.\mathrm{n}=5\right)$. CRP, $\mathrm{C}$-reactive protein; IL, interleukin; $\mathrm{TNF}$, tumor necrosis factor.

to guide the grading of PT. It is one of the most frequently used methods for clinical PT examination. However, it is not suitable for patients with aberrant hemodynamics or contrast agent anaphylaxis, and radiation damage and inconvenient follow-up have limited its further clinical application $(14,15)$. ERCP has become a significant treatment means due to the advantages of being minimally invasive and quick to use with few complications. It not only displays pancreatic duct and biliary ducts, but can also be used for stent implantation and inner drainage. However, ERCP may increase the risk of iatrogenic traumatic pancreatitis $(16,17)$. MRCP has become a preferred diagnostic method for pancreaticobiliary disease due to being noninvasive, lacking radioactive damage potential and not requiring the use of a contrast agent. However, the longer

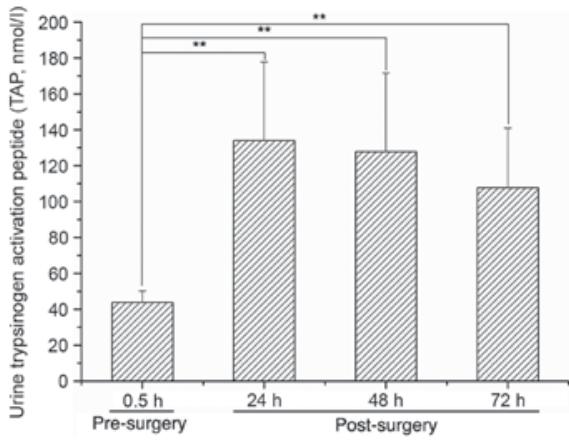

Figure 5. ELISA results for urinary TAP levels. Urinary levels of TAP were increased after pancreatic trauma-inducing surgery, peaking at $24 \mathrm{~h}$ then slightly decreasing at 48 and $72 \mathrm{~h}\left({ }^{* *} \mathrm{P}<0.01\right.$ vs. pre-surgery; $\left.\mathrm{n}=5\right)$. TAP, trypsinogen activation peptide. 
A

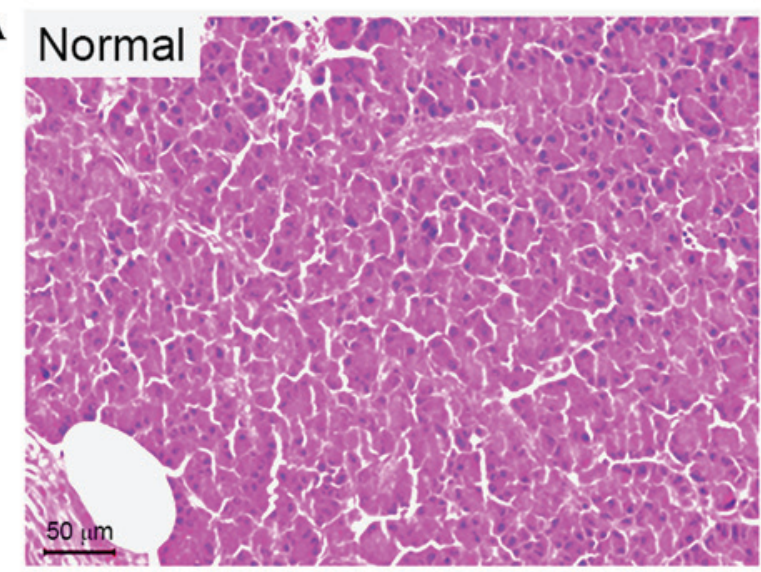

B

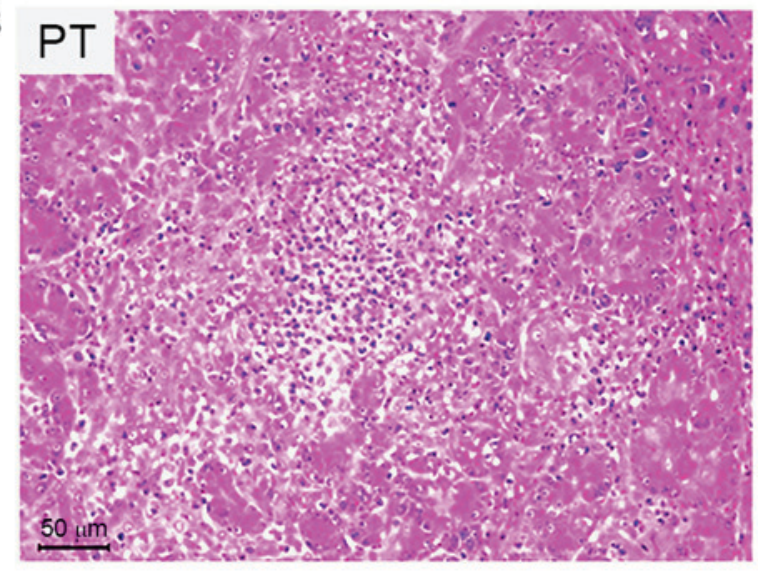

Figure 6. Morphological appearance of pancreatic tissue as revealed by hematoxylin and eosin staining. Morphological appearance of (A) normal pancreatic tissue (B) and pancreatic tissue at $72 \mathrm{~h}$ after pancreatic trauma-inducing surgery. Following the trauma, the pancreatic cells were loosely distributed, with damaged acini, hyperchromatic nuclei and severe inflammatory cell invasion. PT, pancreatic trauma.

time it takes to perform and its high expense have limited its application in PT $(18,19)$. Ultrasound has a significant application value in emergency medicine due to its characteristics of providing real-time images rapidly, safely, inexpensively and conveniently. It enables the early examination of ascites, and repeated observation of their changes can help to verify the state of the illness. However, the sensitivity of ultrasound is relatively low for the diagnosis of PT (20).

CEUS utilizes the nonlinear characteristics and backscattering of blood gas microbubbles to obtain contrast-enhanced images $(21,22)$. In CEUS, contrast agent microbubbles as a blood tracer are injected intravenously to display the blood perfusion characteristics of normal and abnormal tissues. This technique, which is widely used in clinical imaging examinations, has the characteristics of practicability, noninvasiveness and convenience, and has wide applicability for the diagnosis of abdominal parenchymal organ trauma, such as that affecting the liver, spleen and kidney. Over the past 20 years, we have enacted CEUS examination of the liver, spleen and kidney, and significantly increased the trauma diagnostic rate (23-25). In addition, CEUS examination of PT has attracted considerable attention, and demonstrated increasing relevance for PT foci $(26,27)$. In the present study, routine ultrasound and CEUS were selected for the observation and evaluation of the Beagle model of grade III PT. After simple preparation of the intestinal cavity, the display rate of the pancreas by routine ultrasound was $100 \%$. At 24, 48 and $72 \mathrm{~h}$ after the modeling procedure, the PT focus could be clearly displayed, and manifested an irregular area of low to no echo, with a depth greater than half of the anteroposterior diameter of the pancreas, and a blurred edge to the low echo area. CEUS clearly revealed disruption of the pancreatic capsule, the edge and scope of the trauma focus, trauma to tissues surrounding the pancreas and active bleeding. The trauma focus manifested no perfusion or a little low enhancement at the arterial and venous phases. At the post-surgery follow-up examination, the Beagles did not require narcosis, and could be quickly and noninvasively examined using a bedside machine. All of these observations indicated that CEUS was a preferable image detection method for this Beagle model of grade III PT.

To elucidate the reliability and availability of this PT model, levels of amylase and lipase in the ascites, amylase, lipase,
CRP, IL-6 and TNF- $\alpha$ levels in the serum, and TAP levels in the urine were examined by ELISA. Additionally, pancreatic pathology was examined by H\&E staining. At 24, 48 and $72 \mathrm{~h}$ after modeling, in comparison with their pre-surgery values, all of the aforementioned indices were increased, peaking at 24-48 $\mathrm{h}$ after modeling, and decreased thereafter. Among these, urinary TAP levels exhibited significant differences at 24, 48 and $72 \mathrm{~h}$ after modeling in comparison with the pre-surgery level, which indicated that urine TAP may be a sensitive test index for grade III PT in Beagles. All animals developed ascites, with elevated levels of amylase and lipase, which were $>3$-fold higher than the normal reference value and indicated that trauma of the pancreatic parenchyma and pancreatic duct led to pancreatic fluid spillover. No inflammatory response or bleeding necrosis were evident in the abdominal wall and duodenum suture, which indicated that the suture and fixation methods used created little damage, as well as being easy to use. The trauma resulted in damage to the pancreatic acinar cells, with interstitial edema, blood extravasation and inflammatory cell infiltration. This indicates that the grade III Beagle model of PT was correctly established. However, this study also has certain limitations, such as the small sample size, risk of infection following laparotomy and short-term observation period, and therefore the future studies should increase the sample size and prolong the observation time to confirm the veracity and effectiveness.

\section{Acknowledgements}

The present study was supported by the National Natural Science Foundation of China (grant no. 81327003).

\section{References}

1. Lv F, Tang J, Luo Y, Nie Y, Liang T, Jiao Z, Zhu Z and Li T: Emergency contrast-enhanced ultrasonography for pancreatic injuries in blunt abdominal trauma. Radiol Med 119: 920-927, 2014.

2. Cirillo RL Jr and Koniaris LG: Detecting blunt pancreatic injuries. J Gastrointest Surg 6: 587-598, 2002.

3. Toro A, Cavallaro A, Mannino M, Cappello G, Politi A and Di Carlo I: Pancreatic injury in a blunt abdominal trauma treated by a conservative approach with Tachosil ${ }^{\circledR}$. Minerva Chir 67: 461-463, 2012. 
4. Heuer M, Hussmann B, Lefering R, Taeger G, Kaiser GM, Paul A and Lendemans S; Trauma Registry of the DGU: Pancreatic injury in 284 patients with severe abdominal trauma: Outcome, course, and treatment algorithm. Langenbecks Arch Surg 396: 1067-1076, 2011

5. Hasanovic J, Agic M, Rifatbegovic Z, Mehmedovic Z and Jakubovic-Cickusic A: Pancreatic injury in blunt abdominal trauma. Med Arch 69: 130-132, 2015.

6. Goh E and Chen EM: Traumatic pancreatic transection from blunt abdominal trauma. CJEM 16: 502-503, 2014

7. Rui-Wu D, Guang-Yu C, Fa-Qun H, Zu H, Hong-Tao Y, Hong-Yin L, Tao W, Ning L, Li-Jun T and Li-Ping C: Cell cycle characteristics of the pancreas in an animal model of isolated pancreatic trauma. J Trauma Acute Care Surg 76: 784-790, 2014

8. Rosen M, Walsh RM and Goldblum JR: Application of a new collagen-based sealant for the treatment of pancreatic injury. Surg Laparosc Endosc Percutan Tech 14: 181-185, 2004.

9. Song Q, Tang J, Lv FQ, Zhang Y, Jiao ZY, Liu Q and Luo YK: Evaluation of blunt pancreatic injury with contrast-enhanced ultrasonography in comparison with contrast-enhanced computed tomography. Exp Ther Med 5: 1461-1465, 2013.

10. Zhou P, Wang L, Huang S, Fu C, He H, Hong M, Su S and Li S Beagle dogs have low susceptibility to BJ94-like H9N2 avian influenza virus. Infect Genet Evol 31: 216-220, 2015

11. Moore EE, Gogbil TH, Malangoni MA, Jurkovich GJ, Champion HR, Gennarelli TA, McAninch JW, Pachter HL, Shackford SR and Trafton PG: Organ injury scaling II: Pancreas, duodenum, small bowel, colon, and rectum. J Trauma 30 : $1427-1429,1990$

12. Gao H, Song Q, Lv F, Wang S, Wang Y, Li X, Luo Y, Mei X and Tang J: Protection provided by a gabexate mesylate thermo-sensitive in situ gel for rats with grade III pancreatic trauma. Gut and liver 11: 156-163, 2017.

13. Teh SH, Sheppard BC, Mullins RJ, Schreiber MA and Mayberry JC: Diagnosis and management of blunt pancreatic ductal injury in the era of high-resolution computed axial tomography. Am J Surg 193: 641-643, 2007.

14. Zhang G, Yang ZG, Yao J, Deng W, Zhang S, Xu HY and Long QH: Differentiation between tuberculosis and leukemia in abdominal and pelvic lymph nodes: Evaluation with contrast-enhanced multidetector computed tomography. Clinics (Sao Paulo) 70: 162-168, 2015.

15. Tal S, Pollak L and Berkovitz N: Contrast-enhanced computed tomography as a necessary scan in acute stroke: A case series. J Stroke Cerebrovasc Dis 24: 1548-1554, 2015.

16. An JS, Moon SH, Chun SY, Kim JH, Koh DH, Yoon JH and Jeon TY: Value of CT for ERCP endoscopists to identify the type of gastroenteric anastomosis in patients with previous subtotal gastrectomy. Hepatogastroenterology 61: 916-919, 2014.
17. Finkelmeier F, Tal A, Ajouaou M, Filmann N, Zeuzem S, Waidmann $\mathrm{O}$ and Albert J: ERCP in elderly patients: Increased risk of sedation adverse events but low frequency of post-ERCP pancreatitis. Gastrointest Endosc 82: 1051-1059, 2015.

18. Petrescu I, Bratu AM, Petrescu S, Popa BV, Cristian D and Burcos T: CT vs. MRCP in choledocholithiasis jaundice. J Med Life 8: 226-231, 2015.

19. Botsford A, McKay K, Hartery A and Hapgood C: MRCP imaging of duplicate gallbladder: A case report and review of the literature. Surg Radiol Anat 37: 425-429, 2015.

20. McGahan JP, Horton S, Gerscovich EO, Gillen M, Richards JR, Cronan MS, Brock JM, Battistella F, Wisner DH and Holmes JF: Appearance of solid organ injury with contrast-enhanced sonography in blunt abdominal trauma: Preliminary experience. AJR Am J Roentgenol 187: 658-666, 2006.

21. Yuan MX, Li R, Zhang XH, Tang CL, Guo YL, Guo DY and Luo MK: Factors affecting the enhancement patterns of intrahepatic cholangiocarcinoma (ICC) on contrast-enhanced ultrasound (CEUS) and their pathological correlations in patients with a single lesion. Ultraschall Med 37: 609-618, 2016.

22. Sessa B, Trinci M, Ianniello S, Menichini G, Galluzzo M and Miele V: Blunt abdominal trauma: Role of contrast-enhanced ultrasound (CEUS) in the detection and staging of abdominal traumatic lesions compared to US and CE-MDCT. Radiol Med 120: 180-189, 2015.

23. Lv F, Tang J, Li W, Zhang H, Wang W and Yang L: Hemostatic agents injected directly into hepatic injury sites for liver trauma hemorrhage under the guidance of contrast-enhanced ultrasound: An animal experiment. Ultrasound Med Biol 34: 1604-1609, 2008.

24. Tang J, Lv F, Li W, Zhang H, Luo Y, An L and Li T: Percutaneous injection of hemostatic agents for severe blunt hepatic trauma: An experimental study. Eur Radiol 18: 2848-2853, 2008.

25. Lv F, Tang J, Luo Y, Nie Y, Jiao Z, Li T and Zhou X: Percutaneous treatment of blunt hepatic and splenic trauma under contrast-enhanced ultrasound guidance. Clin Imaging 36: 191-198, 2012.

26. Xu HX, Weskott HP, Liu JB and Zheng RQ: Contrast-enhanced ultrasound. Biomed Res Int 2015: 865028, 2015.

27. Meloni MF, Smolock A, Cantisani V, Bezzi M, D'Ambrosio F, Proiti M, Lee F, Aiani L, Calliada F and Ferraioli G: Contrast enhanced ultrasound in the evaluation and percutaneous treatment of hepatic and renal tumors. Eur J Radiol 84: 1666-1674, 2015.

This work is licensed under a Creative Commons

Attribution-NonCommercial-NoDerivatives 4.0

International (CC BY-NC-ND 4.0) License. 